Rupkatha Journal on Interdisciplinary Studies in Humanities (ISSN 0975-2935), Vol. 10, No. 1, 2018 Special Issue on "Interrogating Cultural Translation: Literature and Fine Arts in Translation and Adaptation" In collaboration with the Department of English, Amrita Vishwa Vidyapeetham Guest-edited by Dr. Hari M G, Amrita Vishwa Vidyapeetham, Coimbatore, India

DOI: https://dx.doi.org/10.21659/rupkatha.v10n1.13

Full Text: http://rupkatha.com/V10/n1/v10n113.pdf

\title{
Integrating Translation in Classroom: Facilitating Language Skills
}

\author{
T. Asha Priya ${ }^{1} \&$ B. Jayasridevi ${ }^{2}$ \\ ${ }^{1}$ Associate Professor, Department of English/SCD, SNS College of Engineering. \\ Email: ashapriya20o1@gmail.com \\ ${ }^{2}$ Assistant Lecturer, Department of English/SCD, SNS College of Engineering
}

Received September 27, 2017; Revised December 11, 2017; Accepted December 30, 2017; Published February 04, 2018.

\begin{abstract}
This paper aims at studying the effectiveness of using Grammar Translation method to teach English as a Second Language and formulating recommendations to use this method. It was observed that translation as a method when applied to language teaching practice induced a deeper insight into achieving the desired outcome of teaching. The drawback felt in this particular analysis was because of multi-lingual and diversified classrooms (students with gender and intellectual differences). Translation was not given importance to in second or foreign language classrooms all these years because it was deliberated to be misleading and restraining, discouraging students from thinking in terms of the foreign language. Translation was misinterpreted as one of the many deficient teaching methodologies of olden times. However, in the last few decades, there has been an increasing interest in translation practice in ESL classrooms. Translation, an educational instrument when it is incorporated into the language teaching practice with learning activities, (reading, listening, writing, and vocabulary development) fulfils the purpose of teaching a language. Translation activities like two sides of a coin make students communicate from the Source Language to the Target Language and vice versa. The exercise of translation encourages students to discern the differences in structure and vocabulary, to strengthen their grammatical competence and comprehending ability, to shape their own way of thinking and to correct common mistakes that could otherwise go about unnoticed, thereby helping them to enhance their reading and writing skills, during the process of translation. Translation skills if used properly by students can lead to better understanding and learning of L1.
\end{abstract}

Keywords: Integrated teaching, ESL classrooms, translation, teaching-learning process, language skills

The conventional 'Translation' method first made its appearance into the Second Language (SL) classroom in 188os. According to Prator and Celce-Murcia (1979), the salient features of the Grammar Translation Method are: Classes are taught in the mother tongue, with little active use of the target language; much vocabulary is taught in the form of lists of isolated words; long elaborate explanations of the intricacies of grammar are given; grammar provides the rules for putting words together, and instruction often focuses on the form and inflection of words; little attention is paid to the content of texts, which are treated as exercises in grammatical analysis;

(C) Authors. Published by AesthetixMS under a Creative Commons Attribution Non-Commercial 4.0 International License (http://creativecommons.org/licenses/by-nc/4.0/). 
often the only drills are exercises in translating disconnected sentences from the target language into the mother tongue; little or no attention is given to pronunciation. In the early 150os, teachers used Latin for teaching English. From then on, this method of teaching was employed but after the language died out, it was studied purely as an academic discipline. Slowly, when teachers started teaching other foreign languages in the 19th century, the same translationbased approach crept into the educational system in other countries also.

\section{Literature review:}

The translation method came under heavy criticism in favour of other methods that displaced the use of translation and advocated the use of the 'natural' and 'communicative' approach to language learning. Malmkjaer (1998: 6) briefly summarises the objections to the use of translation in SL classes as follows: (a) Translation is independent of the four skills and radically different from the four skills which define language competence (b) Translation takes up valuable time (c) Translation is unnatural (d) Translation misleads students into thinking that expressions in two languages correspond to one-to-one (e) Translation prevents students from thinking in the foreign language (f) Translation produces interference (g) Translation is a bad test of language skills (h) Translation is only appropriate for the training of translators.

While the English-speaking world has inclined to shun away from the GT method since the middle of the twentieth century, Schjoldager (2004) points out that in some areas of the world, translation continues to be widely used in the language classroom. Translation is not seen as a harmful tool but rather as a pedagogical tool in language learning. Alan Duff, in his book Translation writes that translating authentic texts for a purpose is a communicative activity. He explains how it is more natural and necessary than many of the activities invented for language learners. The pragmatic dimension of translation invites all kinds of discussions; there is no such thing as perfect translation. In addition to written exercises, oral exercises can also be prepared for pair, group and class work.

Nigel J. Ross, who dons the role of a Lexicographer, Translator, Teacher trainer, Language consultant, and Writer claims that 'the real usefulness of translation in the EFL classroom lies in exploiting it in order to compare grammar, vocabulary, word order and other language points in English and the students' mother tongue' (2000, 61).

Translation has a role to play within a broadly defined communicative approach as a means of enabling students to create multi-media texts in both L1 and L2 that communicate in powerful and authentic ways with multiple audiences. Kirsten M Hummel in his "Translation and Short-term L2 Vocabulary Retention: Hindrance or Help?" reiterates the role that active translation may have in enhancing Second Language vocabulary. For this, he has studied three methods- L1 to L2, L2-L1 and rote copying task and "a significant advantage was found for the rote-copying condition compared to the two translation conditions". (Feb. 2010) Gonzalez-Davies (2004, p. 3) points out that concepts that are central to the Communicative Approach, such as learner autonomy, peer work, meaningful learning, learning to learn, decision making and student-centred classes are all concepts that are relevant to translation training as well. She claims that translation assignments can be designed to enable students to develop a number of competencies, including reading and writing skills (Gonzalez-Davies, 2004, p.2). Butzcamm and Caldwell (2009, p.197) argue that translation should be regarded as a fifth skill, whose efficacy for communicative development has been disparaged in the past. Further evidence to reinstate TILT is found in a study conducted by Brooks-Lewis (2009), which reports on students' resistance to a 
monolingual classroom and challenges the theory and practice of the exclusion of the learners' L1 in the classroom. Cook (2013) points to a growing literature which supports a return to bilingual teaching for a number of acquisitioned, pedagogic, political and educational reasons.

Semantic, pragmatic and communicative aspects of language are channelized towards pedagogical efforts more than purely the linguistic aspects. The process does not involve teaching language as a discipline but rather provides the learner with academic knowledge and as a tool of effective communication. That is the reason why more and more linguistic-driven approaches of language teaching fuse with the functional approach, and thus advocate learning grammar and other linguistic aspects only in the service of a communicative competence. This quest has enabled us to take the GT method of teaching English. If translation is serving this purpose of learning a language, why not use the method effectively to teach ESL?

\section{Research Methodology}

The Researchers conducted an experimental research in classrooms through a series of activities. The experiment was planned and executed especially for students who have communication difficulties, who shun away from the confident English speakers, and also those who are from the Tamil medium of instruction. These students come after class hours to improve their proficiency. These students were interested in exercising extra efforts to improve their English.

\section{Research objectives:}

1. To study the impact of Grammar Translation Method

2. To analyze the effectiveness of GT method by using classroom activities

3. To formulate recommendations to use Grammar Translation method while teaching English as a Second Language

Population: SNS College of Engineering, Kurumbapalayam, Coimbatore, Tamil Nadu, India.

Sampling: Students who were interested in learning Everyday English were selected from the first year and second year classes.

Sample size: The sample consists of 35 students.

Tool: A worksheet consisted of classroom activities was designed. (ANNEXURE)

\section{Experiment:}

Bearing in mind all the benefits and limitations of GT method, the researchers proposed to conduct an experiment in the classrooms. The investigation and the results obtained from the analysis aims on proving that translation is an effective tool.

\section{Analysis}

Short story telling is a powerful and motivating source for teaching the four skills - listening, speaking, reading and writing. The use of simplified text with less proficient readers is highly suggested for the sake of matching the level of the students with that of the texts. 
The short story that serves the purpose of this experiment is In a Forest, A Deer, written by Ambai and translated by Lakshmi Holmstorm. Reading the short stories of both Ambai and her translator Lakshmi Holmstrom was a pleasure, and it opened up new vistas of ideas to be used for teaching. It is felt that such a text would be suitable for the experiment.

In classrooms, short stories written in Tamil by Ambai and their translations done by Lakshmi Holstrom have been used extensively. The reading of the text in the original language was done as a part of an off-hand exercise (pre-reading) and the reading aloud of the translated text was done in the class (during reading). This 'round robin reading' enhances the students' listening skills and of course their reading and pronunciation. The students comment on this was that in the beginning they found it difficult to concentrate on the listening, some pronunciations were different, and those who read also had their shortcomings. For them, when they read, some parts of texts like articles and prepositions had gone astray. And for some, reading aloud had long been forgotten (done only in lower classes). So, they found it strange to concentrate and read. But gradually, they are tuned to this exercise of reading aloud, fine tooth comb to minute details like singular - plural words, spellings, punctuation marks, articles, prepositions, conjunctions, and sentence structure. Comprehension abilities have also moved stealthily into the students' consciousness. The outcome of this strenuous reading exercise was that some students have now turned to take reading books as their hobbies and as a result there has been an increase in the number of student entries in the reading Club of the college. Also there are upcoming book reviews from the Reading Club members. They have written book reviews on books Study in Scarlet, The Adventure of Black Peter, The Adventure of the Empty House. Academically too, the students' mastery in comprehending has started outdoing. And their understanding has considerably increased. This has helped the students not only to understand and comprehend second language easily and effectively, but also to provide them with the first-hand experience of a foreign tongue and an exercise to have a better understanding of original works. Thus, the strategies as advocated by Harvey \& Goudvis, namely: thinking, problem solving and monitoring understanding have been achieved.

Reading or studying translated books, articles or other materials also make it easy to remember enormous grammatical rules and numerous vocabulary lists. (Paul Kaye, n.d) Furthermore, through a contrastive study of the target language with the native language, students are given an insight into the linguistic structure and the linguistic phenomenon of two languages. Obviously, experiencing translation or reading translated texts not only does enrich one's mind, but also helps to develop a positive attitude towards the society and also towards foreign cultures.

The functional element of translational practice was mainly designed by the researchers with the aim of developing speaking /communicative aspects of the students. To cater to the need of the hour, students were given more attention/focus on this aspect. A variety of methods of evaluation and assessment like the following:

Describing a character from the story - the students were asked to choose a character (by picking up lots) and described the character for one or two minutes. They took characters like Thangam Athai, Valli, etc. The best characterisation was accolade. This boosted their confidence level and their focus on minute details.

Interviewing the main character - Thangam Athai, this exercise focussed on the conversational practise and helped the students to confront with their everyday formal and informal conversational difficulties. It improves the delivery style, tone, stress, pause, intonation and presentation skills. One of the main disadvantages of the regional medium students is the co- 
relation between their thought process and mode of expression; this is gradually improved upon.

Giving a different ending to a story - such an activity enhances the creativity, imagination, logical and reasoning expertise, social and emotional balance. In addition, the ending gives a variant adaptable to the present cultural scenario and it caters to the present needs of the society.

The different endings tried by the students edged on the idea of adoption, test tube babies, etc.

Group discussion on the main theme - the students' general awareness, sharing of thoughts, confronting the criticism positively, divergent solutions to the same problem, speaking to the point not beating about the bush, to be on track, body language, were checked and made to reflect upon. These act as reality checkers for placement procedure, project presentations, viva voice, etc.

Role play - to be in somebody's shoes - improves the students' understanding of others points of view, their own individuality, dramatic and theatre skills, emotional stability, tackling one's own and others' mood swings.

Such activities were planned and carried out meticulously to improvise speaking skills.

Analysing about this, the students felt that a lot of importance was given to this skill. They found that many words matched the actual words in their mother tongue and that they had difficulty in choosing the usage of the correct word while expressing and that it was challenging to arrive at the feelings and emotions of expression. The various activities that aimed on communication in classrooms in English motivated students to speak continuously in English, encouraged them to participate in paper presentations, poster presentations, and gradually promoted easy and fluent communication.

During translation, which is also working on parallel texts, it was possible to discover the role played by a grammatical rule and how it is actually applied. Some students had a special difficulty in identifying the right tenses especially, when it came to perfect forms, and translating them correctly. Furthermore, when it came to preparing questions and answers, there was a difficulty in placing the words in proper order. For example, "Where are you coming from?" when translated in Tamil writes “நீ எங்கிருந்து வருகிறாய்?” In such translation, where the sentence pattern of the target language and the second language differed, the students were given extra exercises to ease out the learning process. Later, simple activities that focused on identifying the different patterns of speeches, identifying the phrasal verbs, idioms and phrases and understanding their meanings and finally reproducing them in sentences of their own were done with ease by the students.

In addition to the three major skills, writing was also concentrated upon, but not on a larger scale. There were simple exercises involving translation of words and translation of simple passages to and from the Li and the target language. Commenting on this, the students felt that it was rather difficult in analysing and getting the exact matching word or expression. The discussion of an entire translated passage or even of one word in the classroom taught the students that a word usually does not have just one possible translation. Students learn that every word assumes a different meaning according to the context. In this regard, students were of the opinion that they found it surprising that many words were English words that were unconsciously used by them in their regular conversation. (such words as night, stars, forest, deer) But, when it came to translating from L1 to L2 or L2 to L1 they had to spend a lot of time and 
effort in completing the task, especially when the translation had cultural differences. For example, translating the sentence, "அப்படீன்னா அவங்க பெரியவளே ஆகலை" என்றாள். This has more of cultural and gender connotaions.

Although our students do not intend to enter the translation profession, for them translating helps to relate L1 to L2: a mental process that takes place in their minds on an unconscious level, every time they speak the other language.

As a first approach, it is useful to translate short sentences in order to be able to build a longer paragraph and deepen the structure of the single phrase later on. A word-for-word backtranslation enables the student to highlight the relationship between the two languages. L1 and L2 have different structures. Back-translation involves mainly the syntactical structure, rather than only the lexical level; it is a comparison between the patterns of two languages where individual lexical units may or may not match. It is possible to understand the sentence on a logical level and consequently convey the meaning in the L2. This exercise entails interpreting a text and the awareness that losses, gains, compensations, omissions and shifts often occur in translation. Parallel translation is not always possible, not only for reasons of grammar, but also for sociocultural reasons. A free translation becomes a useful tool to point out aspects of a culture, and consequently to master a language.

Vocabulary was concentrated and workouts like matching the Tamil words with their English equivalents, adding prefixes and suffixes to the root words were done with ease by the students as they had the dictionary to help them. The students responded by saying that the worksheet put to light the power of developing their vocabulary and the usefulness of being a logophile.

While translation as a meaningful and helpful means is widely used in ESL classrooms and has been creating benefits to language learners, some concerns were also raised by the researchers (Luo, 2007; Richards \& Rodgers, 2001). They suggest that translation should be limitedly used in the process of language acquisition because translation used as a teaching method reverses the language learning process (Luo, 2007; Richards \& Rodgers, 2001). It can be seen that the longstanding use of translation in ESL classrooms limits the proficiency of speaking skills. Linguistic researchers claim that using translation to teach another language fails to establish a direct link between thoughts and expressions in target languages (Richards \& Rodgers, 2001). Translation forces students to think and express their opinions in native languages instead of target languages and makes it difficult for those who want to give up the habit of organizing speeches in native languages and then translating the ideas into foreign languages (Malmkjaer, 1998). As a result, language learners who are taught with this method often fail to express themselves effectively in target languages as well as to get proficiency in its actual application. This is what some educationalists have criticised, but from the proposed experiment and analysis of results, it is found that students are hassle free to express themselves and there has been improvement in their learning period and the teachers also feel that the language skills have been enhanced by using the Grammar-Translation method.

\section{Conclusion}

As with all experiments, this also poses a few limitations. Apart from the one mentioned above, the one that we experienced was that the classrooms were of a heterogeneous one. The students belonged to different categories and they learnt at different speeds. The quick learners actually 
found some activities very easy and hence a lazy, lethargic attitude was displayed. In contrast to this the achievers (as it is called for slow learners) were very enthusiastic in their approach towards these exercises. The gender also mattered here, because the main theme dealt with the problem faced by an infertile woman. The words used to describe her infertility are better understood by girl students rather than boys, and further there is an inhibition for girls to discuss matters with their counterparts. Here, freedom of expression was inhibited. And certain words needed extra attention for the exact matching and no two translations were the same.

Translation as an exercise involves various aspects including cultural backgrounds, individual comprehensions and language competency. These factors require translators not only to possess a profound knowledge of the source language to perceive the meaning in different contexts, but also to have the ability to recreate the messages in the target language precisely. Besides, translation also concerns linguistic, textual and social systems that are related to both languages and cultures. Therefore, language teachers should enrich their knowledge in both their mother tongue and the target language, as well as improve their language competence including translation skills to achieve an effective and successful language teaching objective. Translating cannot be separated from interpreting, even when we speak about a written text. The purpose of both is to transfer information from the ST to the TT. Interpreting can serve as a mental exercise to improve the students' speaking skills, although they will still need to practice conversation.

The effective worth of the translation methods when employed deftly in ESL classrooms holds several themes: (1) translation as a method can take advantage of the students' instinctive ability to interpret and assimilate L2 where he gained the information through L1 processing, (2) the usage of bilingual immersion, co-teaching, bi-lingual text usage or a combination of different approaches, a teacher helps the student to gain confidence, and increases his security, (3) he is able to distinguish the differences and find the similarities of both the languages in terms of its structure, usage, specific and general vocabulary, and the order of presentation of its information. The exercise itself is an exciting experience in language learning process.

\section{Note about the Short Story Writers:}

Lakshmi Holmström MBE is an Indian-born British writer, literary critic and translator of Tamil fiction and poetry into English. Her translations include Fish in a Dwindling Lake, a translation of short stories by Ambai (Penguin India, 2012); A Second Sunrise: Poems by Cheran, translated and edited by Lakshmi Holmström \& Sascha Ebeling (Navayana, 2012). In 2000, she received the Crossword Book Award for her translation of Karukku by Bama (OUP, 2012); in 2007 she shared the Crossword-Hutch Award for her translation of Ambai's short stories, In a Forest, a Deer; in 2008, she received the Iyal Award from the Tamil Literary Garden, Canada.

The beauty of Lakshmi's translation is that the words retain from the original shine with the pride of having retained their position. Some words are such; they're better off being left without the burden of taking on a flat, 'transliteration'. For instance, how else does one describe the 'chombu'? - Lakshmi Holstrom keeps the word in her translation of Ambai's novella Wrestling. Lakshmi remarks in her Translator's note in 'In a Forest, A Deer', that to translate Ambai is a privilege and a pleasure, but her work also presents many challenges. "One such challenge is the complex form of some of her short stories -or novellas -, rather - which use different perspectives and voices, and interweave different sort of texts" (2006). 


\section{References}

Ambai, alias C.S. Lakshmi. காட்டில் ஒரு மான்.

Alan, Duff. Translation. ELBS with Oxford University Press, 1989.

Butzkamm, W. \& Caldwell, J.A.W. (2009). The Bilingual Reform: A Paradigm Shift in Foreign Language Teaching. Tubingen: Narr Studienbucher.

Cook, G. (2009). Use of Translation in Language Teaching. In M. Baker \& G. Saldanha (Eds.), Routledge Encyclopedia of Translation Studies. Second Edition (pp. 112-115). New York/London: Routledge.

Gonzalez-Davies, M. (2004). Multiple Voices in the Translation Classroom: Activities, tasks and projects. Amsterdam: John Benjamens.

Holmstorm, Lakshmi. In a Forest, A Deer. OUP (2000).

Harvey, S. \& Goudvis, A. (2000). Strategies that work: Teaching Comprehension to Enhance Understanding. Markham: Pembroke

Kelly, Niamhand Jennifer Bruen. Translation as a pedagogical tool in the foreign language classroom: A qualitative study of attitudes and behaviours._Version 1.2. July 2016

Kirsten M Hummel. "Translation and Short-term L2 Vocabulary Retention: Hindrance or Help?" Language Teaching Research, Vol. 14, 1: pp. 61-74., February 3, 2010.

Krashen, S. D., \& Terrell, T. D. (1998). The Natural Approach: Language Acquisition in the Classroom. Prentice Hall International.

Leonardi, Vanessa. "The Role of Pedagogical Translation in Second Language Acquisition: From Theory to Practice". International Academic Publishers. Bern, Switzerland. 200o: 61.

Malmkjær, K. Introduction: Translation and Language Teaching: Language Teaching and Translation. Manchester: St. Jerome Publishing.1998

Prator, Clifford H. \& Celce-Murcia, Marianne, 1979: "An outline of language teaching approaches." In Celce-Murcia, Marianne \& McIntosh, Lois (Ed.), "Teaching English as a Second or Foreign Language," Newbury House.

Richards, Jack. C and Theodre S. Rodgers. Approaches and Methods in Language Teaching. Sec. Edn. Cambridge University Press. 2001.

Ross, J. Nigel. “Interference and Intervention: Translation in the EFL Classroom”. Modern English Teacher, 2000.

Schjoldager, A. (2004). “Are L2 learners more prone to err when they translate?" In Malmkjær, K., \& McEnery, A.E. (Eds.), Translation in Undergraduate Degree Programmes (pp. 127-14). Amsterdam: John Benjamins Publishing Company.

Seong Man Park. The Use of Students' First Language in the EFL Classroom in Korea. Humanizing Language Teaching. Year 15; Issue 2; April 2013, ISSN 1755-9715

https://www.researchgate.net/publication/274496636_Translation_as_a_pedagogical_tool_in_the_foreign_l anguage_classroom_A_qualitative_study_of_attitudes_and_behaviours [accessed May 29, 2017].

https://www.teachingenglish.org.uk/article/translation-activities-language-classroom 


\section{ANNEXURE - WORKSHEET}

I. Match the Tamil phrases in column A with its relevant English phrase in column B:

S No.

1

2

3

4

5
COLUMN A

புரையில்வைத்தபடி

வெளிப்படையாக

உயிர்ப்பிக்கும்

பிழைக்கவைத்து

அணைத்தவாறு

II. Parts of speech test

A. Provide the adverbs of the words given:

\section{COLUMN B}

D. within a niche

E. overtly discernible

B. vitalising

A. resuscitate

Croak

Discern

Press

Perform

Emerge

B. Now provide the nouns for the same set of words.

Croak

Discern

Press

Perform

Emerge

C. Adjectives

Croak

Discern

Press

Perform

Emerge

D. Use the appropriate Prefix or Suffix or both to the root words given in the text to make meaningful words. Type the prefixed word for every root word with a '-' between them.

NOTE: All root words do not necessarily have both suffixed and prefixed words.

E. Add the part of speech after the word.

comprehend 
Clasp

Sudden

Happy

Gleam

F. Identify and write the idioms or phrasal verbs and its appropriate meaning.

made up

came by

Steeped in

nooks and crannies

G. Look up the meaning of these words in the dictionary and make sentences of your own, using those words.

Nudged

Encased

threshold

III. Translate the following sentences into English without changing its essence in your words.

1. வள்ளி தாவணி போட்டவள். "அப்படீன்னா அவங்க பெரியவளே ஆகலை" என்றாள்.

2. தங்கம் அத்தை தான் கதை சொல்வாள்.

3. அவளே இட்டுக் கட்டியவை. 